\title{
CARTOGRAFIADO GEOLÓGICO DE COSTA RICA EN LA REVISTA GEOLÓGICA DE AMÉRICA CENTRAL
}

\author{
GEOLOGICAL MAPPING OF COSTA RICA IN THE CENTRAL AMERICAN \\ JOURNAL OF GEOLOGY
}

\author{
Percy Denyer
Escuela Centroamericana de Geología, Universidad de Costa Rica. Apdo. 214-2060 UCR. Costa Rica- América Central percy.denyer@ucr.ac.cr

(Recibido: 15/09/2014; aceptado: 10/10/2014)

\begin{abstract}
The geological map is a model of reality, in which the geological units are grouped by a classification that follows international standards. The first geological maps of Costa Rica were made in the late XIX century and early XX century. The geological map is not an end in itself, but a tool for understanding the geological history of a region and the processes of erosion and weathering that have affected existing rocks. There are four basic types of geological maps: geological reconnaissance maps, regional geological maps, detailed geological maps, and specialized maps. Regional maps are some of the most important because their scale, 1:50 000 or 1: 25000 , is suitable for use as a basis for various academic and economic studies as well as for planning purposes.

The Central American Journal of Geology has contributed to the distribution and publication of geological maps, with a total coverage of $8638 \mathrm{~km} 2$, corresponding to $17 \%$ of the national territory. In the future, the magazine is fully prepared to continue the dissemination of cartographic information.

Keywords: Costa Rica, geological maps, mapping, scale, coordinates.

RESUMEN: El mapa geológico es un modelo de la realidad, en el cual se agrupan las unidades geológicas mediante una clasificación que sigue parámetros internacionales. Los primeros mapas geológicos de Costa Rica fueron hechos a finales del siglo XIX e inicios del siglo XX. El mapa geológico no es un fin en sí mismo, sino que es un instrumento para entender la historia geológica de una región y los procesos de erosión y meteorización que afectaron las rocas existentes. Existen cuatro tipos básicos de mapas geológicos: mapas de reconocimiento, regionales, detallados y especializados. Los mapas regionales son uno de los más importantes, ya que por su escala 1:50 000 o 1: 25 000, son usados como base para diferentes estudios académicos y de índole económico, así como para efectos de planificación. La Revista Geológica de América Central ha contribuido con la distribución y publicación de mapas geológicos, con un total de $8638 \mathrm{~km} 2$, que corresponde con un $17 \%$ del territorio nacional. En el futuro, la revista está en plena disposición de continuar con la difusión de la información cartográfica.

Palabras clave: Costa Rica, mapa geológico, cartografiado, escala, coordenadas.
\end{abstract}




\section{INTRODUCCIÓN}

La corteza de la Tierra es muy delgada al compararla con el radio total del planeta, y de esto, lo que es posible observar sobre la superficie terrestre, son solo pequeños fragmentos de una realidad escondida tras suelos, vegetación y estructuras antropogénicas. De estos "retazos", debe hacer el geólogo sus interpretaciones, que lo llevan a hacer un modelo de la realidad, el mapa geológico.

El mapa geológico representa entonces un registro de la información geológica, tal como la distribución y naturaleza de las rocas, las cuales se agrupan y clasifican en unidades fundamentales, lo que representa una "taxonomía" que agrupa, ordena, sistematiza y jerarquiza las rocas, lo cual se hace según las leyes básicas de la ciencia geológica y los lineamientos expresados en las normas estratigráficas oficiales de la International Union of Geological Sciences (Salvador, 1994; Barragán et al., 2010). Las unidades geológicas están morfológicamente controladas por estructuras que las contornean, cortan y traslapan, lo que da forma al paisaje de la superficie terrestre y que, por lo tanto, forman parte fundamental del mapa geológico.

El primer mapa geológico detallado de una parte de Costa Rica fue elaborado por William More Gabb y data de 1873 (Denyer \& Soto, 2000), en este mapa se cubría el área de Talamanca, desde la ciudad de Limón hasta la frontera con Panamá y partes de la cordillera de Talamanca.

La primera versión de un mapa geológico que cubre todo el territorio de Costa Rica fue publicado en 1905 por Karl T. Sapper (Alvarado \& Denyer, 2012). Posteriormente destacan el mapa escala 1: 750000 de la Dirección de Geología, Minas y Petróleo (1963) y el mapa escala 1: 700 000 de Dóndoli (1968). Mapas más detallados a escala 1: 200000 son publicados en 1982 (MIEM, 1982). Después de esto, se publican ediciones a una escala menos detallada (1: 500 000) por Tournon \& Alvarado (1997), posteriormente a escala 1: 750 000 por Recope (1997) y finalmente Denyer \& Alvarado (2007), a una escala 1: 400000.

\section{El significado del mapa geológico}

El mapa geológico no es un fin en sí mismo, como a veces podría mal entenderse, sino que es un instrumento para explicar la geología de un área, su historia geológica y los procesos de erosión y meteorización involucrados, así como los procesos tectónicos que han contribuido a deformar la región. Todos estos factores han dado como resultado un intrincado juego de formas que se expresan los mapas topográficos.

Por lo tanto, el mapa geológico es el punto de partida para los diferentes estudios específicos, tanto de índole científico como tecnológico. $\mathrm{Su}$ importancia trasciende el conocimiento puro, pues proporciona las ubicaciones y distribución de los diferentes tipos de roca, que eventualmente serán fuentes de materiales para la construcción de obras civiles, pilar de los índices económicos del país. También aporta parte de los datos fundamentales de tipo tectónico y geodinámica externa, insumo fundamental para desarrollar los planes de emergencia en cuanto a la amenaza geológica; y son un instrumento esencial para la definición de los planes reguladores cantonales.

\section{EL MAPA GEOLÓGICO}

\section{La escala}

La escala en un mapa topográfico es un factor muy importante que determina la correspondencia de una unidad de medida en el mapa, p.ej. 1 $\mathrm{cm}$, con la distancia correspondiente en la realidad (el terreno). Es así como un mapa 1: 10000 implica que $1 \mathrm{~cm}$ en el mapa corresponde con 100 $\mathrm{m}$ en el terreno, y así sucesivamente, una escala 1: 50000 implica que $2 \mathrm{~cm}$ en el mapa son $2 \mathrm{~km}$ en el terreno. Por lo tanto, una escala 1: 10000 va a ser mucho más grande que una escala 1: 50000 .

Otra implicación de la escala es la densidad de curvas de nivel. En Costa Rica, por ejemplo, los mapas publicados por el Instituto Geográfico Nacional cumplen con características tales como que los mapas 1: 200000 tienen curvas de nivel 
cada $100 \mathrm{~m}$, mientras que los de escala 1: 50000 tienen una mayor densidad de curvas (cada $20 \mathrm{~m}$ ). O sea, que el detalle topográfico es muchísimo mayor en un mapa 1: 50000 que en uno 1: 200 000. El mayor o menor detalle es evidente en el cartografiado de lagunas o el ancho de los ríos.

El mapa geológico ha sido levantado sobre una base topográfica, por lo que el grado de detalle que este tenga la geología es dependiente del mapa topográfico. La escala de un mapa geológico va mucho más allá del tamaño de publicación, tiene que ver con el detalle con que se ha hecho el cartografiado y con su posible utilización.

Actualmente es indispensable el uso de una escala gráfica, tanto en mapas formales como en los esquemas que acompañan las publicaciones. Esto debido a la reproducción en fotocopias y digitalización de la información.

\section{Mantener el concepto de escala}

Actualmente, con el uso de los Sistemas de Información Geográfica (SIG), los cambios de escala son muy sencillos de realizar, lo que causa que haya una mala utilización del concepto de escala. En realidad, el o los autores de un mapa deben dar validez a la utilización de una escala al considerar la información disponible y el detalle suministrado. Por lo tanto, los mapas pueden pasarse a una escala menor, pero nunca deberían pasarse a una escala mayor, pues esto involucra la sobreestimación de información.

\section{Escogencia del tipo de coordenadas}

En Costa Rica se ha usado por años un sistema de coordenada cartográfica, usando una proyección conformal cónica en que divide al país en las proyecciones Lambert Norte y Lambert Sur. A partir de junio del 2007, el sistema de referencia oficial es el CRTM05 con base en el decreto No. 33797-MJ-MOPT, que fue publicado en el diario La Gaceta No. 108 (Fallas, 2011). Sin embargo, el
Instituto Geográfico Nacional (IGN), no ha publicado los mapas con las respectivas coordenadas, por lo que el uso del nuevo sistema de referencia no es práctico para el trabajo geológico. Por lo tanto el uso de Lambert Norte y Sur es el sistema más utilizado hasta la fecha.

\section{TIPOS DE MAPA GEOLÓGICO}

Lisle et al. (2011) consideran que existen 4 tipos de mapa geológico, que a continuación se describirán:

\section{Mapa de reconocimiento}

Es aquel mapa que se hace de una forma rápida, cuya escala es de 1: 250000 o menor. Muchas veces se basan únicamente en sensores remotos y las corroboraciones de campo son muy pocas. Se obtienen datos muy generales sobre estructuras regionales.

\section{Mapa regional}

Muestra un mayor detalle geológico, aunque normalmente se usan herramientas de sensores remotos como fotos aéreas, DEMs, imágenes satelitales, con un amplio reconocimiento de unidades en el campo y sus resultados se expresan generalmente en escalas 1: 50000 o 1: 25000 . Podría considerarse que requiere de puntos de observación cada kilómetro, sin embargo esto es muy relativo, depende de: las vías de acceso, la diversidad de litologías, la distribución de las estructuras geológicas, el control geomorfológico de las unidades, las condiciones topográficas, la complejidad geológica o tectónica de algunas unidades específicas, y otros muchos factores que harán que los encargados del proyecto dediquen más o menos recorridos a ciertas zonas del mapa.

Requiere de un conocimiento y exposición de la columna estratigráfica, así como de las estructuras tectónicas principales. Se indican los datos de la inclinación y dirección estratigráfica de 
estratos, diques, etc. Normalmente se incluye un perfil geológico, que es un elemento que añade la tercera dimensión al mapa y que deja traslucir el modelo geológico del área.

Son mapas de tipo conceptual, donde lo más importante no es la exactitud de los contactos y las trazas de estructuras, sino que las relaciones espaciales de las unidades correspondan con la columna estratigráfica y que las estructuras muestren un concepto estructural regional.

Este tipo de mapas son muy útiles para ser utilizados por los tomadores de decisiones acerca de la ubicación de proyectos, así como la planificación del uso del terreno. Son la base para la elaboración de mapas más detallados o específicos, los cuales normalmente mantienen las relaciones estratigráficas y las estructuras regionales.

\section{Mapa detallado}

Son mapas realizados en investigaciones específicas, sean de tipo científico o tecnológico, generalmente a escalas de 1: 10000 o mayores. Muy comunes en el estudio de mineralizaciones, represas hidroeléctricas, o cualquier proyecto ingenieril.

\section{Mapa especializado}

Son de características muy variadas, tanto para investigación como con propósitos económicos, generalmente su escala varía entre 1 : 10000 y 1: 2500, aunque pueden ser de mayor escala. Pueden ser mapas de gran escala de pequeñas áreas para dilucidar el comportamiento de estructuras geológicas, planos de minas subterráneas, con escalas mayores a 1: 1500. Hay muchos otros tipos de mapas con afiliación geológica como sería el caso de mapas de levantamientos geofísicos o geoquímicos. Actualmente están acompañados de una base de datos del Sistema de Posicionamiento Global (GPS por sus siglas en inglés), lo que hace a estos mapas muy versátiles y que da la posibilidad de sobreponerlos a los datos de geología convencional, para hacer interpretaciones y correlaciones espaciales de los datos.

\section{DESCRIPCIÓN DE UNIDADES}

Se considera, como principio básico, que cualquier cartografiado debe basarse en criterios claros de reconocimiento en el campo de las unidades geológicas De esta forma, las unidades geológicas tendrán una mayor utilización práctica, tanto para investigaciones científicas como para su utilización en proyectos civiles y de planificación.

En Costa Rica, al igual que en el resto de América Central, no existe una Comisión Estratigráfica, que se encargue de la regulación y establecimiento de unidades geológicas. Por lo tanto, hasta el momento ha funcionado de forma intuitiva por la costumbre del uso y desuso de nomenclaturas, así como el criterio subjetivo de los autores y revisores de artículos, como es el caso de la Revista Geológica de América Central (RGAC).

En pro de una estandarización de criterios, se considera que deben utilizarse los criterios publicados respecto a las descripciones, criterios de reconocimiento, descripción, jerarquía y cambios del Código Estratigráfico Internacional Norteamericano (Salvador 1994 y Barragán, 2010).

Dentro de la descripción de unidades debe considerarse la inclusión de al menos los siguientes ítems, principalmente en el caso de descripción de nuevas unidades:

- Estratotipos y otros estándares de referencia

- Descripción en la localidad tipo

- Aspectos regionales 
- $\quad$ Edad

- Correlación con otras unidades y otras áreas

- Génesis

- Antecedentes

\section{SIMBOLOGÍA}

La simbología es un elemento fundamental en un mapa geológico. Sin embargo, no hay reglas internacionales definidas al respecto y además existen particularidades de cada área a las que la simbología debe adaptarse. Por lo tanto, se considera que lo más importante es que exista una lista de símbolos específicos para cada mapa.

Existe un listado de símbolos, que utiliza el United States Geological Survey (USGS), que puede ser muy útil en una publicación del USGS (Federal Geographic Data Committee, 2006).

Una serie de patrones litológicos cuyo significado es de fácil comprensión y que pueden ser utilizados en programas comunes como Adobe Illustrator, facilita la comprensión de los mapas e ilustraciones geológicas. Se pueden encontrar los archivos en el cibersitio: https://blogs.otago. ac.nz/si-geology/resources/illustrationgraphicsresources/usgs-ai-pack-instructions-and-download-link/

\section{EL TIEMPO GEOLÓGICO}

La Comisión Internacional de Estratigrafía (International Commission on Stratigraphy) publica constantemente cambios a la Tabla del Tiempo Geológico, la última versión 2014/02 se puede encontrar en el cibersitio: www.stratigraphy.org.

La Tabla del Tiempo Geológico representa también una escala cronoestratigráfica de colores que debería ser usada para representar las diferentes unidades geológicas por su edad. Sin embargo, en una región como Costa Rica, con una gran geodiversidad en una escala temporal muy corta, se hace imposible restringirse a esta escala pues hay muchas unidades y se necesitan más colores para ser representadas debidamente.

\section{EL ROL DE LA RGAC EN LA CARTOGRAFÍA GEOLÓGICA DE COSTA RICA}

Debido a la necesidad de un cartografiado geológico sistemático de Costa Rica, la RGAC ha estimulado la publicación y distribución de los mapas geológicos. Primeramente se distribuyeron mapas publicados por el IGN, a escala 1: 50 000, en las revistas 11, 12 y 16 (Cuadro 1), en ocasiones en que los textos explicativos correspondían con una publicación de la RGAC.

Posteriormente, se inició la serie: Colección de Mapas Geológicos, que publicó en los números 28, 29, 30, 32, 33, 40 y 41 (Cuadro 1) mapas geológicos escala 1: 100 000. Estos se publicaron a color, en las páginas finales de la RGAC, en hoja doble carta.

En términos comparativos, las contribuciones de la RGAC corresponden a $8638 \mathrm{~km}^{2}$, que corresponde con un $17 \%$ del territorio nacional. $\mathrm{La}$ distribución es mayor en la zona de Guanacaste, la región central y el pacífico central (Fig. 1).

\section{¿QUÉ SE ESPERA PARA EL FUTURO?}

La necesidad del cartografiado es imperante pues el faltante en mapas es todavía muy grande. Así que, desde este punto de vista, la RGAC está dispuesta a seguir con la publicación de los mapas geológicos. Sin embargo, en los últimos años se han visto iniciativas de la Dirección de Geología y Minas del Ministerio de Ambiente y Energía para la publicación de mapas a escala 1: 50 000, que se reinició con un convenio con el Servicio Geológico Checo. A partir de este convenio, se 


\section{Cuadro 1}

Mapas geológicos distribuidos y publicados en 30 años de la RGAC

\begin{tabular}{|c|c|c|c|c|c|}
\hline Mapa & Nombre & Área $\left(\mathrm{km}^{2}\right)$ & Escala & $\mathrm{N}^{0}$ revista & Autores \\
\hline 1 & $\begin{array}{c}\text { Coyolito y Punta } \\
\text { Morales }\end{array}$ & 136 & $1: 25000$ & 11 & Denyer (1990) \\
\hline 2 & Río Grande & 510 & 1: 50000 & 12 & Arias \& Denyer (1991) \\
\hline 3 & Candelaria & 510 & 1: 50000 & 12 & Denyer \& Arias (1991) \\
\hline 4 & Caraigres & 510 & 1: 50000 & 12 & Arias \& Denyer (1991) \\
\hline 5 & Abra & 510 & 1: 50000 & 12 & Denyer \& Arias (1991) \\
\hline 6 & Carrillo Norte & 395 & 1: 100000 & 16 & Arias \& Denyer (1993) \\
\hline 7 & Belén & 510 & 1: 100000 & 16 & Denyer \& Arias (1993) \\
\hline 8 & Matapalo - Punta Gorda & 159 & 1: 100000 & 16 & Arias \& Denyer (1993) \\
\hline 9 & Matambú & 510 & 1: 100000 & 28 & Denyer \& Flores (2003) \\
\hline 10 & Talolinga & 510 & 1: 100000 & 28 & Flores \& Denyer (2003) \\
\hline 11 & Barranca & 510 & 1: 100000 & 29 & Denyer et al. (2003) \\
\hline 12 & Abangares & 510 & 1: 100000 & 29 & Flores et al. (2003) \\
\hline 13 & Cañas & 510 & 1: 100000 & 30 & Aiazzi et al. (2004) \\
\hline 14 & Golfo & 126 & 1: 100000 & 32 & Denyer et al. (2005) \\
\hline 15 & Berrugate & 172 & 1: 100000 & 32 & Flores \& Denyer (2005) \\
\hline 16 & Tierras Morenas & 510 & 1: 100000 & 33 & Civelli et al. (2005) \\
\hline 17 & General & 510 & 1: 100000 & 40 & Alvarado et al. (2009) \\
\hline 18 & San Isidro & 510 & 1: 100000 & 40 & Alvarado et al. (2009) \\
\hline 19 & Fortuna & 510 & 1: 100000 & 41 & Alvarado (2009) \\
\hline 20 & Buenos Aires & 510 & $1: 100000$ & 41 & Obando \& Kussmaul (2009) \\
\hline
\end{tabular}

publicaron las hojas: Juntas, Chapernal y Miramar (Žáček et al., 2010a, 2010 b y 2010c) donde la RGAC tuvo a su cargo la publicación del texto de la hoja Miramar (Žáček, 2012). Posteriormente se publicó la hoja Naranjo (Huapaya \& Rojas, 2012). Por último, en el 2013, en un proyecto de cooperación UCR-MINAET, se publican una serie de
21 hojas topográficas (Denyer et al., 2013), con un texto que lo acompaña (Denyer et al., 2014).

Por lo anterior, se espera que se siga con una campaña tendiente a llegar a tener la cartografía completa del país a escala 1: 50 000. Para tal fin, la RGAC está a disposición de colaborar con la publicación de los textos y los mapas. 


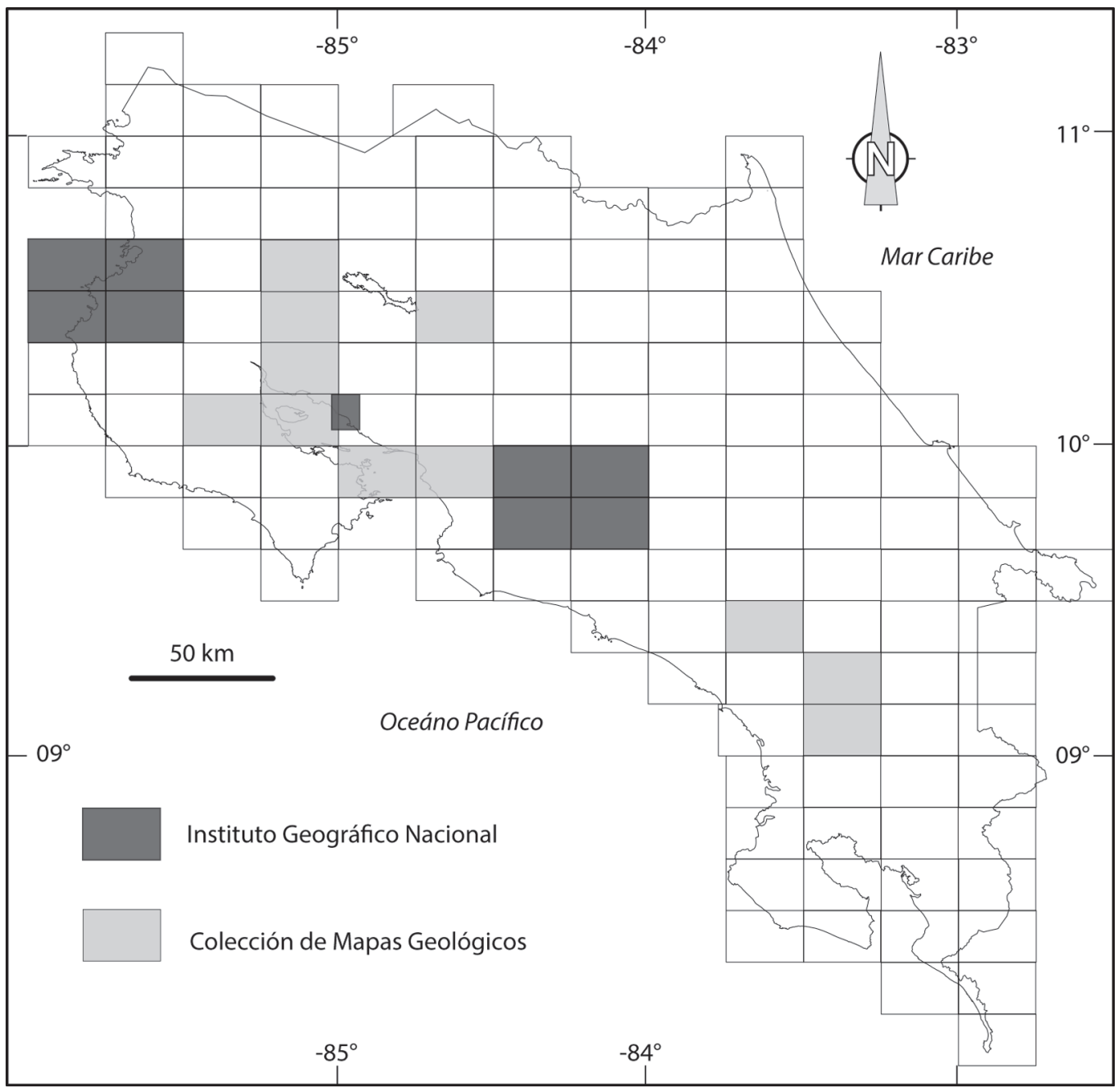

Fig. 1: Mapas geológicos publicados como parte de los artículos de la Revista Geológica de América Central. Incluye aquellos mapas publicados en la Sección: Colección de Mapas Geológicos (Escala 1: 100 000), así como los mapas publicados por el Instituto Geográfico Nacional y distribuidos como parte de la revista (1:50 000). 


\section{REFERENCIAS BIBLIOGRÁFICAS}

AIAZZI, D., FIORETTA, M., CIVELLI, G., CHIESA, S. \& ALVARADO, G.E., 2004: Geología de la hoja Cañas.Escala 1: 100 000, Rev. Geól. Amér. Central, 30.

ALVARADO, G. E., 2009: Mapa geológico de la hoja Fortuna .- Escala 1:100 000, Rev. Geól. Amér. Central, 41.

ALVARADO, G. E. \& DENYER, P., 2012: Karl T. Sapper (1866-1945): geólogo pionero en América Central.- 232 págs. + 4 mapas. San José. Editorial UCR.

ALVARADO, G. E., TAYLOR, W.,BARQUERO, R., LÓPEZ, A., CERDAS, A. \& MURILLO, J., 2009: Mapa geológico de la hoja General.- Escala 1: 100 000, Rev. Geól. Amér. Central, 40.

ALVARADO, G. E., BARQUERO, R., TAYLOR, MORA, M., PERALDO, P., SALAZAR, G. \& AGUILAR, T., 2009: Mapa Geológico de la hoja San Isidro.- Escala 1: 100 000, Rev. Geól. Amér. Central, 40.

ARIAS, O. \& DENYER, P., 1990: Geología de la hoja Caraigres (mapa 1:50 000).- San José: Instituto Geográfico Nacional.

ARIAS, O. \& DENYER, P., 1990: Geología de la hoja Río Grande (mapa 1:50 000).- San José: Instituto Geográfico Nacional.

ARIAS, O. \& DENYER, P., 1992: Mapa geológico de la hoja Carrillo Norte (1:50 000).San José: Instituto Geográfico Nacional.

ARIAS, O. \& DENYER, P., 1992: Mapa geológico de las hojas Matapalo y Punta Gorda (1:50 000).- Inst. Geogr. Nac., San José.
BARRAGÁN, R., CAMPOS-MADRIGAL, E., FERRUSQUÍA-VILLAFRANCA, I., LÓPEZ-PALOMINO, I. \& TOLSON, G., 2010: Código Estratigráfico Norteamericano.- 48 págs. Univ. Nac. Autónoma de México, México D.F.

CIVELLI, G., LOCATI, U., BIGIOGGERO, B., CHIESA, S., ALVARADO, G. E. \& MORA, G. P., 2005: Mapa geológico de la hoja Tierras Morenas.- Escala 1:100 000, Rev. Geól. Amér. Central, 33.

DENYER, P. (Ed.), 1988: Mapa geológico Punta Morales-Coyolito-Manzanillo (1:25 000).Inst. Geogr. Nac., San José.

DENYER, P. \& ALVARADO, G. E., 2007: Mapa Geológico de Costa Rica.- Escala 1: 400 000, Oficializado por la Dirección de Geología y Minas. Librería Francesa, San José, Costa Rica.

DENYER, P. \& ARIAS, O., 1990: Geología de la hoja Abra (mapa 1:50 000).- Inst. Geogr. Nac., San José.

DENYER, P. \& ARIAS, O., 1990: Geología de la hoja Candelaria (mapa 1:50 000).- Inst. Geogr. Nac., San José.

DENYER, P. \& ARIAS, O., 1992: Mapa geológico de la hoja Belén (1:50 000).- Inst. Geogr. Nac., San José.

DENYER, P. \& SOTO, G., 2000: Análisis de los trabajos de William M. Gabb sobre Costa Rica, a la luz del paradigma geológico del siglo XIX.- Rev. Geól. Amér. Central, 23: 97-118.

DENYER, P., AGUILAR, T. \& MONTERO, W., 2014: Cartografiado geológico de la península de Nicoya: Estratigrafía y Tectónica.208 págs. Ed. UCR, San José. 
DENYER, P., AGUILAR, A. \& ALVARADO, G.A. 2004: Mapa Geológico de la Hoja Barranca .- Colección de Mapas Geológicos (3). Rev. Geol. Amér. Central29.

DENYER, P., MONTERO, W. \& FLORES, K., 2004: Mapa Geológico de la Hoja Golfo.Colección de Mapas Geológicos (6). Rev. Geol. Amér. Central, 32.

DENYER, P., AGUILAR, T. \& MONTERO, W., 2013: Mapa geológico de la hoja Abangares.- Escala 1: 50 000, CICG-UCR \& DGM-MINAE, San José.

DENYER, P., AGUILAR, T. \& MONTERO, W., 2013: Mapa geológico de la hoja Belén.Escala 1: 50 000, CICG-UCR \& DGMMINAE, San José.

DENYER, P., AGUILAR, T. \& MONTERO, W., 2013: Mapa geológico de la hoja Berrugate.- Escala 1: 50 000, CICG-UCR \& DGM-MINAE, San José.

DENYER, P., AGUILAR, T. \& MONTERO, W., 2013: Mapa geológico de la hoja Cabuya.Escala 1: 50 000, CICG-UCR \& DGMMINAE, San José.

DENYER, P., AGUILAR, T. \& MONTERO, W., 2013: Mapa geológico de la hoja Carrillo Norte.- Escala 1: 50 000, CICG-UCR \& DGM-MINAE, San José.

DENYER, P., AGUILAR, T. \& MONTERO, W., 2013: Mapa geológico de la hoja Cerro Azul.- Escala 1: 50 000, CICG-UCR \& DGM-MINAE, San José.

DENYER, P., AGUILAR, T. \& MONTERO, W., 2013: Mapa geológico de la hoja Cerro Brujo.- Escala 1: 50 000, CICG-UCR \& DGM-MINAE, San José.
DENYER, P., AGUILAR, T. \& MONTERO, W., 2013: Mapa geológico de la hoja Diriá.Escala 1: 50 000, CICG-UCR \& DGMMINAE, San José.

DENYER, P., AGUILAR, T. \& MONTERO, W., 2013: Mapa geológico de la hoja Garza.Escala 1: 50 000, CICG-UCR \& DGMMINAE, San José.

DENYER, P., AGUILAR, T. \& MONTERO, W., 2013: Mapa geológico de la hoja Golfo.Escala 1: 50 000, CICG-UCR \& DGMMINAE, San José.

DENYER, P., AGUILAR, T. \& MONTERO, W., 2013: Mapa geológico de la hoja Marbella.- Escala 1: 50 000, CICG-UCR \& DGM-MINAE, San José.

DENYER, P., AGUILAR, T. \& MONTERO, W., 2013: Mapa geológico de la hoja Matambú.- Escala 1: 50 000, CICG-UCR \& DGM-MINAE, San José.

DENYER, P., AGUILAR, T. \& MONTERO, W., 2013: Mapa geológico de la hoja Matapalo.- Escala 1: 50 000, CICG-UCR \& DGM-MINAE, San José.

DENYER, P., AGUILAR, T. \& MONTERO, W., 2013: Mapa geológico de la hoja Puerto Coyote.- Escala 1: 50 000, CICG-UCR \& DGM-MINAE, San José.

DENYER, P., AGUILAR, T. \& MONTERO, W., 2013: Mapa geológico de la hoja Punta Gorda.- Escala 1: 50 000, CICG-UCR \& DGM-MINAE, San José.

DENYER, P., AGUILAR, T. \& MONTERO, W., 2013: Mapa geológico de la hoja Río Arío.- Escala 1: 50 000, CICG-UCR \& DGM-MINAE, San José. 
DENYER, P., AGUILAR, T. \& MONTERO, W., 2013: Mapa geológico de la hoja Talolinga.- Escala 1: 50 000, CICG-UCR \& DGM-MINAE, San José.

DENYER, P., AGUILAR, T. \& MONTERO, W., 2013: Mapa geológico de la hoja Tempisque.- Escala 1: 50 000, CICG-UCR \& DGM-MINAE, San José.

DENYER, P., AGUILAR, T. \& MONTERO, W., 2013: Mapa geológico de la hoja Tambor.Escala 1: 50 000, CICG-UCR \& DGMMINAE, San José.

DENYER, P., AGUILAR, T. \& MONTERO, W., 2013: Mapa geológico de la hoja Venado.Escala 1: 50 000, CICG-UCR \& DGMMINAE, San José.

DENYER, P., AGUILAR, T. \& MONTERO, W., 2013: Mapa geológico de la hoja Villarreal.- Escala 1: 50 000, CICG-UCR \& DGM-MINAE, San José.

DEPARTAMENTO DE GEOLOGÍA, MINAS Y PETRÓLEO, 1963: Mapa geológico de Costa Rica.- Escala 1: 750 000, Ministerio de Industrias, San José.

DÓNDOLI, C., 1968: Mapa Geológico de Costa Rica.- Escala 1: 700 000, Dirección de Geología, Minas y Petróleo, Ministerio de Industria y Comercio, San José.

FALLAS, J., 2011: Sistemas de Información Geográfica: fuentes de geodatos para Costa Rica.- 60 págs. Universidad Nacional, Escuela de Ciencias Ambientales, Heredia.
FEDERAL GEOGRAPHIC DATA COMMITEE, 2006: Digital Cartographic Standard for Geologic Map Symbolization.- 290 págs. USGS, Reston, Virginia.

FLORES, K., DENYER, P \& AGUILAR, T. 2004: Mapa Geológico de la Hoja Abangares.Colección de Mapas Geológicos (4). Rev. Geol. Amér. Central, 29.

FLORES, K. \& DENYER, P., 2004: Mapa geológico de la Hoja Berrugate.- Colección de Mapas Geológicos (7). Rev. Geol. Amér. Central, 32.

HUAPAYA, S. \& ROJAS, V., 2012: Mapa geológico de la hoja Naranjo (2012).- Escala 1: 50 000. Dirección de Geología y Minas, San José.

LISLE, R.J., BRABJAM, P.J. \& BARNES, J.W., 2011: Basic Geological Mapping [5th edition].- 217 págs. Willey Blackwell, West Sussex UK.

MIEM (Ministerio de Industria, Economía y Comercio), 1982: Mapa Geológico de C. R.- 9 mapas escala 1:200 000. S Inst. Geogr. Nac., San José..

OBANDO, G. \& KUSSMAUL, S., 2009: Mapa geológico de la hoja Buenos Aires.- Escala 1:100 000, Rev. Geól. Amér. Central, 41.

RECOPE, 1987: Mapa geológico de C. R.- Escala 1: 750 000. Inst. Geogr. Nac., San José.

SALVADOR, A, 1994: International Stratigrahpic Guide.- 214 págs. The International Union of Geological Sciences, Boulder-Colorado. 
TOURNON, J. \& ALVARADO, G. E. 1995: Mapa Geológico de Costa Rica.- Escala 1: 500000. Ministtere de Affaires Étrangeres- Instituto Costarricense de Electricidad, Paris.

ŽÁČEK,V., VOREL, T., KYCL, P. \& HUAPAYA, S., 2010a: Mapa geológico de la hoja Miramar.- Escala 1:50 000, Serv. Geol. Checo, Praga.

ŽÁČEK, V., ČECH, S., HAVLÍČEK, P., VOREL, T., DUDÍK SCHULMANNOVÁ, B., KYCL, P. \& HUAPAYA, S., 2010b: Mapa geológico de la hoja Chapernal.- Escala 1:50 000, Serv. Geol. Checo, Praga.
ŽÁČEK, V., ČECH, S., DUDÍK SCHULMANNOVÁ, B., VOREL, T., KYCL, P., HUAPAYA, S., 2010c: Mapa geológico de la hoja Las Juntas.Escala 1:50 000, Serv. Geol. Checo, Praga.

ŽÁČEK, V., VOREL, T. KYCL, P., HUAPAYA, S., MIXA, P., GRYGAR, R., HAVLÍČEK, P., ČECH, S., HRAZDÍRA, P., METELKA, V., ŠEVČ́́K, J. \& PÉCSKAY, Z., 2012: Geología y estratigrafía de la Hoja 3646-II Miramar, Costa Rica.- Rev. Geol. Amér. Central, 47: 7-54. 
